Article

\title{
In Vitro Characterization of Multidrug-Resistant Influenza A(H1N1)pdm09 Viruses Carrying a Dual Neuraminidase Mutation Isolated from Immunocompromised Patients
}

\author{
Emi Takashita ${ }^{1, * \mathbb{D}}$, Seiichiro Fujisaki ${ }^{1}$, Masaru Yokoyama ${ }^{2} \mathbb{D}$, Masayuki Shirakura ${ }^{1}$, \\ Hiroko Morita ${ }^{1}$, Kazuya Nakamura ${ }^{1}$, Noriko Kishida ${ }^{1}$, Tomoko Kuwahara ${ }^{1}$, Hironori Sato ${ }^{2}$, \\ Ikuko Doi $^{3}$, Yuji Sato ${ }^{4}$, Shinichi Takao ${ }^{5}$, Yukie Shimazu ${ }^{5}$, Takeshi Shimomura ${ }^{6}$, Takuo Ito ${ }^{7}$, \\ Shinji Watanabe ${ }^{1}$, Takato Odagiri ${ }^{1}$ and on behalf of The Influenza Virus Surveillance Group \\ of Japan ${ }^{+}$ \\ 1 Influenza Virus Research Center, National Institute of Infectious Diseases, Tokyo 208-0011, Japan; \\ seifuji@nih.go.jp (S.F.); masas@nih.go.jp (M.S.); h-morita@nih.go.jp (H.M.); kazuyan@nih.go.jp (K.N.); \\ kishidan@nih.go.jp (N.K.); kuwahara@nih.go.jp (T.K.); sw@nih.go.jp (S.W.); todagiri@nih.go.jp (T.O.) \\ 2 Pathogen Genomics Center, National Institute of Infectious Diseases, Tokyo 208-0011, Japan; \\ yokoyama@nih.go.jp (M.Y.); hirosato@nih.go.jp (H.S.) \\ 3 Ibaraki Prefectural Institute of Public Health, Ibaraki 310-0852, Japan; i.doi@pref.ibaraki.lg.jp \\ 4 Tsukuba Memorial Hospital, Ibaraki 300-2622, Japan; satou_yuji@tsukuba-kinen.or.jp \\ 5 Hiroshima Prefectural Technology Research Institute, Hiroshima 734-0007, Japan; \\ s-takaoe1077@pref.hiroshima.lg.jp (S.T.); y-shimazu89236@pref.hiroshima.lg.jp (Y.S.) \\ 6 National Hospital Organization Hiroshimanishi Medical Center, Hiroshima 739-0696, Japan; \\ shimomuratakeshi2010@gmail.com \\ 7 National Hospital Organization Kure Medical Center, Hiroshima 737-0023, Japan; itot@kure-nh.go.jp \\ * Correspondence: emitaka@nih.go.jp \\ + The members of the group are listed in the Acknowledgments.
}

Received: 12 August 2020; Accepted: 31 August 2020; Published: 2 September 2020

\begin{abstract}
Influenza A(H1N1)pdm09 viruses carrying a dual neuraminidase (NA) substitution were isolated from immunocompromised patients after administration of one or more NA inhibitors. These mutant viruses possessed an H275Y/I223R, H275Y/I223K, or H275Y/G147R substitution in their NA and showed enhanced cross-resistance to oseltamivir and peramivir and reduced susceptibility to zanamivir compared to single $\mathrm{H} 275 \mathrm{Y}$ mutant viruses. Baloxavir could be a treatment option against the multidrug-resistant viruses because these dual H275Y mutant viruses showed susceptibility to this drug. The G147R substitution appears to stabilize the NA structure, with the fitness of the H275Y/G147R mutant virus being similar or somewhat better than that of the wild-type virus. Since the multidrug-resistant viruses may be able to transmit between humans, surveillance of these viruses must continue to improve clinical management and to protect public health.
\end{abstract}

Keywords: influenza; neuraminidase inhibitor; oseltamivir; peramivir; zanamivir; laninamivir; baloxavir; favipiravir; resistance

\section{Introduction}

In Japan, four neuraminidase (NA) inhibitors-oseltamivir, peramivir, zanamivir, and laninamivir-and a cap-dependent endonuclease inhibitor, baloxavir marboxil, have been approved for the treatment of influenza [1]. In addition, favipiravir, a viral RNA-dependent RNA polymerase inhibitor, has been approved for influenza pandemic preparedness [2]. Since nationwide 
monitoring is important for public health planning and clinical management, we have been conducting surveillance of antiviral-resistant viruses.

During our surveillance, we detected multidrug-resistant influenza A(H1N1)pdm09 viruses from immunocompromised patients [3-5]. These viruses possessed an I223K, an I223R, or a G147R substitution in combination with an H275Y substitution in their NA protein. Several studies have reported the detection of $\mathrm{A}(\mathrm{H} 1 \mathrm{~N} 1) \mathrm{pdm} 09$ viruses carrying a dual $\mathrm{H} 275 \mathrm{Y}$ substitution, such as H275Y/E119D, H275Y/E119G, H275Y/I223K, H275Y/I223R, and H275Y/S247N, from immunocompromised patients [6-11]. A few studies have been carried out to understand the impact of the H275Y/E119D and H275Y/I223R substitutions on viral fitness [12-14]; however, that of the $\mathrm{H} 275 \mathrm{Y} / \mathrm{I} 223 \mathrm{~K}$ and $\mathrm{H} 275 \mathrm{Y} / \mathrm{G} 147 \mathrm{R}$ substitutions remains unknown. Here, we report our assessment of the in vitro properties of H275Y/I223K, H275Y/I223R, and H275Y/G147R dual mutant viruses isolated from immunocompromised patients.

\section{Materials and Methods}

\subsection{Viruses}

The dual H275Y mutant viruses (H275Y/I223K, H275Y/I223R, and H275Y/G147R) were detected from immunocompromised patients hospitalized in hematology departments after administration of one or more NA inhibitors (Table 1). The representative single H275Y mutant and wild-type viruses detected during the same influenza season are shown in Table 2. These representative viruses are genetically most closely related to the corresponding dual H275Y mutant viruses in the Global Initiative on Sharing All Influenza Data (GISAID) EpiFlu database (http://www.gisaid.org). A/Hiroshima/57/2014, $\mathrm{A} / \mathrm{Osaka} / 8 / 2014$, and A/Sakai/23/2013 have hemagglutinin (HA) genes that belong to genetic clade 6B. A/Ibaraki/54/2016, A/Yokohama/94/2016, and A/Yokohama/40/2016 have HA genes that belong to genetic clade 6B.1. A/Hiroshima/13/2016, A/Aichi/83/2016, and A/Yokohama/59/2016 have HA genes that belong to genetic clade 6B.2. All viruses were propagated in MDCK (NBL-2) cells (ATCC CCL-34). The dual substitutions were confirmed in both clinical specimens and isolates by sequencing them.

Table 1. Immunocompromised patients infected with the dual H275Y mutant influenza $\mathrm{A}(\mathrm{H} 1 \mathrm{~N} 1) \mathrm{pdm} 09$ viruses.

\begin{tabular}{|c|c|c|c|c|}
\hline Isolate Name & $\begin{array}{l}\text { Date of Symptom } \\
\text { Onset } \\
\text { (Day/Month/Year) }\end{array}$ & $\begin{array}{l}\text { Antiviral Administration } \\
\text { (Day/Month/Year) }\end{array}$ & $\begin{array}{l}\text { Date of Specimen } \\
\text { Collection } \\
\text { (Day/Month/Year) }\end{array}$ & NA Substitution \\
\hline A/Hiroshima/57/2014 & 31-03-2014 & $\begin{array}{l}\text { 31-03-2014: Peramivir } \\
\text { 04-04-2014: Laninamivir }\end{array}$ & 04-04-2014 & H275Y/I223R \\
\hline A/lbaraki/54/2016 & 09-02-2016 & $\begin{array}{l}\text { 09-02-2016: Peramivir } \\
\text { 24-02-2016 to 03-03-2016: Peramivir } \\
\text { 26-02-2016 to 03-03-2016: Oseltamivir } \\
\text { 05-03-2016: Laninamivir }\end{array}$ & 07-03-2016 & $\mathrm{H} 275 \mathrm{Y} / \mathrm{I} 223 \mathrm{~K}$ \\
\hline A/Hiroshima/13/2016 & 26-02-2016 & $\begin{array}{l}\text { 23-02-2016: Laninamivir } \\
\text { 26-02-2016 to 01-03-2016: Peramivir } \\
\text { 07-03-2016 to 11-03-2016: Peramivir } \\
\text { 28-03-2016 to 01-04-2016: Peramivir }\end{array}$ & 11-03-2016 & H275Y/G147R \\
\hline
\end{tabular}

NA: neuraminidase. 
Table 2. Antiviral susceptibilities of the dual H275Y mutant influenza A(H1N1)pdm09 viruses.

\begin{tabular}{|c|c|c|c|c|c|c|c|c|}
\hline \multirow[t]{2}{*}{ Isolate Name } & \multirow[t]{2}{*}{ GISAID Isolate ID } & \multirow[t]{2}{*}{ NA Substitution } & \multicolumn{5}{|c|}{ IC $_{50}, \mathrm{nM}$ (Fold-Change ${ }^{1}$ ) } & \multirow{2}{*}{$\begin{array}{c}\begin{array}{c}\mathrm{EC}_{50,}, \mu \mathrm{M} \\
\text { (Fold-Change }^{1} \text { ) }\end{array} \\
\text { Favipiravir }^{4}\end{array}$} \\
\hline & & & Oseltamivir $^{2}$ & Peramivir $^{2}$ & Zanamivir $^{2}$ & Laninamivir $^{2}$ & Baloxavir $^{3}$ & \\
\hline A/Hiroshima/57/2014 & EPI ISL 160499 & H275Y/I223R & $\begin{array}{l}6263.69 \\
(20,000)\end{array}$ & $\begin{array}{c}944.20 \\
(94,000)\end{array}$ & $\begin{array}{l}4.84 \\
(48)\end{array}$ & $\begin{array}{l}4.48 \\
(20)\end{array}$ & $\begin{array}{l}8.34 \\
(2.1)\end{array}$ & $\begin{array}{l}7.99 \\
(1.2)\end{array}$ \\
\hline A/Osaka/8/2014 & EPI ISL 155839 & $\mathrm{H} 275 \mathrm{Y}$ & $\begin{array}{c}173.80 \\
(560)\end{array}$ & $\begin{array}{c}28.25 \\
(2,800)\end{array}$ & $\begin{array}{l}0.03 \\
(0.3)\end{array}$ & $\begin{array}{l}0.14 \\
(0.6)\end{array}$ & $\begin{array}{l}5.10 \\
(1.3)\end{array}$ & $\begin{array}{l}4.76 \\
(0.7)\end{array}$ \\
\hline A/Sakai/23/2013 & EPI ISL 154461 & $\begin{array}{c}\text { None } \\
\text { (wild-type) }\end{array}$ & 0.31 & 0.01 & 0.10 & 0.22 & 4.05 & 6.64 \\
\hline A/Ibaraki/54/2016 & EPI ISL 221789 & H275Y/I223K & $\begin{array}{l}10161.87 \\
(16,000)\end{array}$ & $\begin{array}{l}501.62 \\
(7,200)\end{array}$ & $\begin{array}{l}2.48 \\
(12)\end{array}$ & $\begin{array}{l}1.94 \\
(6.9)\end{array}$ & $\begin{array}{l}7.24 \\
(1.2)\end{array}$ & $\begin{array}{l}10.20 \\
(1.0)\end{array}$ \\
\hline A/Yokohama/94/2016 & EPI ISL 218900 & $\mathrm{H} 275 \mathrm{Y}$ & $\begin{array}{c}466.79 \\
(730)\end{array}$ & $\begin{array}{l}20.88 \\
(300)\end{array}$ & $\begin{array}{l}0.33 \\
(1.6)\end{array}$ & $\begin{array}{l}0.52 \\
(1.9)\end{array}$ & $\begin{array}{l}3.49 \\
(0.6)\end{array}$ & $\begin{array}{c}21.40 \\
(2.1)\end{array}$ \\
\hline A/Yokohama/40/2016 & EPI ISL 217919 & $\begin{array}{c}\text { None } \\
\text { (wild-type) }\end{array}$ & 0.64 & 0.07 & 0.21 & 0.28 & 6.16 & 10.01 \\
\hline A/Hiroshima/13/2016 & EPI ISL 220376 & H275Y/G147R & $\begin{array}{c}1324.62 \\
(1,500)\end{array}$ & $\begin{array}{l}114.14 \\
(1,400)\end{array}$ & $\begin{array}{l}1.56 \\
(4.5)\end{array}$ & $\begin{array}{l}0.37 \\
(0.9)\end{array}$ & $\begin{array}{l}10.59 \\
(1.0)\end{array}$ & $\begin{array}{l}15.13 \\
(1.7)\end{array}$ \\
\hline A/Aichi/83/2016 & EPI ISL 233222 & $\mathrm{H} 275 \mathrm{Y}$ & $\begin{array}{c}364.44 \\
(420)\end{array}$ & $\begin{array}{l}31.93 \\
(400)\end{array}$ & $\begin{array}{l}0.77 \\
(2.2)\end{array}$ & $\begin{array}{l}0.77 \\
(1.9)\end{array}$ & $\begin{array}{l}10.18 \\
(0.9)\end{array}$ & $\begin{array}{l}14.83 \\
(1.7)\end{array}$ \\
\hline A/Yokohama/59/2016 & EPI ISL 217920 & $\begin{array}{c}\text { None } \\
\text { (wild-type) }\end{array}$ & 0.86 & 0.08 & 0.35 & 0.40 & 11.04 & 8.81 \\
\hline
\end{tabular}

GISAID: Global Initiative on Sharing All Influenza Data; NA: neuraminidase; $\mathrm{IC}_{50}: 50 \%$ inhibitory concentration; $\mathrm{EC}_{50}: 50 \%$ effective concentration. ${ }^{1}$ Fold-change in $\mathrm{IC}_{50}$ or $\mathrm{EC}_{50}$ values compared with the wild-type viruses. ${ }^{2} \mathrm{IC}_{50}$ values were determined by using an NA inhibition assay. ${ }^{3} \mathrm{IC}_{50}$ values were determined by using a focus reduction assay. ${ }^{4} \mathrm{EC} \mathrm{C}_{50}$ values were determined by using a cytopathic effect reduction assay. 


\subsection{Antiviral Compounds}

Oseltamivir acid, peramivir trihydrate, and zanamivir hydrate were purchased from Biosynth Carbosynth (Berkshire, UK). Laninamivir was provided by Daiichi Sankyo (Tokyo, Japan). Baloxavir acid was purchased from MedChemExpress (Monmouth Junction, NJ, USA), and favipiravir was purchased from Cayman Chemical (Ann Arbor, MI, USA). Oseltamivir, peramivir, zanamivir, laninamivir, and favipiravir were dissolved in distilled water, and baloxavir was dissolved in dimethyl sulfoxide.

\subsection{NA Inhibition Assay}

NA inhibitor susceptibilities were determined by using a fluorescence-based NA inhibition assay with the NA-Fluor influenza neuraminidase assay kit (Applied Biosystems, Foster City, CA, USA) and 4-MU-NANA substrate (Biosynth Carbosynth). Briefly, diluted viruses were mixed with 20-fold serial dilutions of $125,000 \mathrm{nM}$ oseltamivir, peramivir, zanamivir, or laninamivir and incubated for $20 \mathrm{~min}$ at $37^{\circ} \mathrm{C}$; 4-MU-NANA substrate was then added, and the mixture was incubated for $30 \mathrm{~min}$ at $37^{\circ} \mathrm{C}$. The reaction was stopped by adding $0.12 \mathrm{M} \mathrm{Na}_{2} \mathrm{CO}_{3}$ in $40 \%$ ethanol. The fluorescence of the solution was measured at an excitation wavelength of $355 \mathrm{~nm}$ and an emission wavelength of $460 \mathrm{~nm}$. The results are expressed as $50 \%$ inhibitory concentration $\left(\mathrm{IC}_{50}\right)$ values, which were calculated by using GraphPad Prism (GraphPad Software, San Diego, CA, USA).

\subsection{Focus Reduction Assay}

Baloxavir susceptibilities were determined by using a focus reduction assay as previously described [1] in humanized MDCK cells (i.e., hCK cells), which express high levels of $\alpha 2,6$-sialoglycans and very low levels of $\alpha 2,3$-sialoglycans [15]. hCK cells were kindly provided by Dr. Yoshihiro Kawaoka (University of Wisconsin-Madison). hCK cells in 96-well plates were infected with 1000 focus-forming units (FFU)/well of viruses. Virus adsorption was carried out for $1 \mathrm{~h}$ at $37^{\circ} \mathrm{C}$ and then an equal volume of $1.2 \%$ Avicel RC-581 (DuPont Nutrition USA, Wilmington, DE, USA) in culture medium containing serial dilutions $(0.025-2500 \mathrm{nM})$ of baloxavir was added to each well in triplicate. The cells were incubated for $24 \mathrm{~h}$ at $34{ }^{\circ} \mathrm{C}$ and then fixed with formalin. After the formalin was removed, the cells were immunostained with a mouse monoclonal antibody against influenza A virus nucleoprotein (Merck KGaA, Darmstadt, Germany), followed by a horseradish peroxidase-labeled goat anti-mouse immunoglobulin (SeraCare Life Sciences, Milford, MA, USA). The infected cells were stained with TrueBlue Substrate (SeraCare Life Sciences) and then washed with distilled water. After cell drying, the focus numbers were quantified by using an ImmunoSpot S6 Analyzer, ImmunoCapture software, and BioSpot software (Cellular Technology, Cleveland, OH, USA). The results are expressed as $\mathrm{IC}_{50}$ values.

\subsection{Cytopathic Effect Reduction Assay}

Favipiravir susceptibilities were determined by using a cytopathic effect reduction assay as previously described [2] in AX4 cells, which overexpress $\alpha 2,6$-sialoglycans [16]. AX4 cells were kindly provided by Dr. Yoshihiro Kawaoka. AX4 cells in 96-well plates were infected with viruses at a multiplicity of infection (MOI) of $0.0150 \%$ tissue culture infective dose $\left(\mathrm{TCID}_{50}\right) /$ cell. Virus adsorption was carried out for $1 \mathrm{~h}$ at $37^{\circ} \mathrm{C}$ and then an equal volume of culture medium containing serial dilutions $(0.05-1000 \mathrm{nM})$ of favipiravir was added to each well in triplicate. The cells were incubated for 3-5 days at $34{ }^{\circ} \mathrm{C}$. To determine the extent of the cytopathic effect, the CellTiter-Glo 2.0 Assay reagent (Promega Corporation, Madison, WI, USA) was added and luminescence was measured. The results are expressed as $50 \%$ effective concentration $\left(\mathrm{EC}_{50}\right)$ values, which were calculated by using GraphPad Prism. 


\subsection{Plaque Assay}

Virus titrations were conducted by using a plaque assay as previously described [2] in AX4 cells. AX4 cells in 6-well plates were infected with serial dilutions of viruses in triplicate. Virus adsorption was carried out for $1 \mathrm{~h}$ at $37^{\circ} \mathrm{C}$. After the inoculum was removed, $0.8 \%$ agarose in culture medium was added to each well. The cells were incubated for 3 days at $34^{\circ} \mathrm{C}$ and the plaque numbers were counted.

\subsection{Virus Replication Kinetics In Vitro}

In vitro replication kinetics of the dual H275Y mutant viruses were determined as previously described [17] in AX4 cells. AX4 cells were infected with viruses at an MOI of 0.001 plaque-forming units (PFU)/cell in triplicate. The cells were incubated at $34^{\circ} \mathrm{C}$. The supernatants were harvested at 12 , $24,36,48,60$, and $72 \mathrm{~h}$ post-infection and were subjected to virus titration by using plaque assays.

\subsection{Competitive Virus Replication In Vitro}

The competitive growth capability of each dual H275Y mutant virus with that of the wild-type virus was compared as previously described [18] in AX4 cells. Each dual H275Y mutant virus was coinfected with the corresponding wild-type virus at an MOI of $0.01 \mathrm{PFU} /$ cell in triplicate. The cells were incubated at $34^{\circ} \mathrm{C}$. At 2 days post-infection, the supernatants were subjected to virus titration by using plaque assays and to deep sequencing analysis to determine the relative proportion of each genotype. The viruses were serially passaged 3-4 times at an MOI of $0.01 \mathrm{PFU} / \mathrm{cell}$.

\subsection{Deep Sequencing Analysis}

Deep sequencing analysis was performed as previously described [2]. A cDNA library was prepared from viral RNA by using the NEBNext Ultra RNA Library Prep Kit for Illumina and NEBNext Singleplex Oligos for Illumina (New England Biolabs, Ipswich, MA, USA), followed by purification by using Agencourt AMPure XP (Beckman Coulter, Brea, CA, USA). The library was sequenced by using MiSeq Reagent Kits v2 with MiSeq (Illumina, San Diego, CA, USA). Sequence reads were aligned to the reference sequence of A/California/07/2009(H1N1)pdm09 by using CLC Genomics Workbench 8 (CLC bio, Aarhus, Denmark).

\subsection{Structural Analysis of the NA Protein}

A structure model of the NA protein of each mutant virus was constructed by use of homology modeling and was refined by using Molecular Operating Environment (MOE) (Chemical Computing Group, Montreal, Canada) as previously described [18]. The crystal structure of the A(H1N1)pdm09 virus NA protein (PDB ID 4B7R; resolution, 1.9 A) [19] served as the modeling template. Single-point mutations were generated on the NA model. Ensembles of the protein conformations were generated by using the LowMode MD module in MOE. The average stability changes of the ensembles were calculated by using the Boltzmann distribution. The stability scores $(\Delta \Delta \mathrm{Gs})$ of the structures were obtained through the stability scoring function of the Protein Design application.

\subsection{Statistical Analysis}

Statistical analyses were performed using GraphPad Prism. Statistically significant differences between groups were determined by using an unpaired t-test calculated by fitting a mixed-effects model. $p$ values of $<0.05$ were considered statistically significant.

\section{Results}

\subsection{Immunocompromised Patients Infected with Dual H275Y Mutant Viruses}

The clinical courses of the immunocompromised patients infected with the dual H275Y mutant viruses are shown in Table 1. The first patient, a woman in her late 70s who was infected with the 
H275Y/I223R mutant virus (A/Hiroshima/57/2014), was treated with peramivir (600 mg) on the day of symptom onset. After four days of peramivir treatment, the patient's symptoms had not improved, and she was therefore treated with laninamivir $(20 \mathrm{mg})$. She recovered after laninamivir administration. The second patient, a man in his late 70s who was infected with the H275Y/I223K mutant virus (A/lbaraki/54/2016), was treated with peramivir $(300 \mathrm{mg})$ on the day of symptom onset. His infection persisted for more than two weeks, so he then received combination therapy of peramivir ( $300 \mathrm{mg}$ ) and oseltamivir (75 mg twice daily) for seven days. However, the influenza rapid diagnostic test remained positive. Laninamivir ( $40 \mathrm{mg}$ ) was administered two days after the end of the combination therapy and after six days of laninamivir administration, the rapid diagnostic test was negative. The third patient, a woman in her early 50s who was infected with the H275Y/G147R mutant virus (A/Hiroshima/13/2016), received prophylaxis with laninamivir $(40 \mathrm{mg})$. Three days later, she had onset of illness and was treated with peramivir $(600 \mathrm{mg})$ for five days. Her infection persisted for more than one month and peramivir $(600 \mathrm{mg})$ was administered for three intermittent periods of five days. She developed left lower lobe pneumonia 20 days post-disease onset and alveolar hemorrhage 12 days after. Two days after the end of the third five-day course, she died.

\subsection{Antiviral Susceptibilities of the Dual H275Y Mutant Viruses}

We compared the susceptibility of the dual H275Y mutant viruses (A/Hiroshima/57/2014, $\mathrm{A} / \mathrm{Ibaraki} / 54 / 2016$, and A/Hiroshima/13/2016) with that of the corresponding single H275Y mutant (A/Osaka/8/2014, A/Yokohama/94/2016, and A/Aichi/83/2016) and wild-type (A/Sakai/23/2013, A/Yokohama/40/2016, and A/Yokohama/59/2016) viruses to four NA inhibitors (oseltamivir, peramivir, zanamivir, and laninamivir), a cap-dependent endonuclease inhibitor, baloxavir, and a viral RNA-dependent RNA polymerase inhibitor, favipiravir (Table 2). The dual H275Y mutant viruses are genetically most closely related to the corresponding single H275Y mutant and wild-type viruses. The H275Y/I223R, H275Y/I223K, and H275Y/G147R mutant viruses exhibited enhanced cross-resistance to oseltamivir and peramivir and reduced susceptibility to zanamivir compared to the corresponding single H275Y mutant viruses. The H275Y/I223R and H275Y/I223K mutant viruses, but not the H275Y/G147R mutant virus, showed reduced susceptibility to laninamivir. All viruses tested were susceptible to baloxavir and favipiravir.

\subsection{In Vitro Replication Kinetics of the Dual H275Y Mutant Viruses}

The impact of the dual substitution on viral growth was assessed in AX4 cells, which overexpress the human influenza receptor ( $\alpha 2,6$-sialoglycans) (Figure 1). Since each of the wild-type viruses has different genetic backgrounds, the virus titers of the wild-type viruses cannot be compared directly with each other. The virus titers of the H275Y/I223R mutant virus were found to be comparable to those of the corresponding wild-type virus as previously described [13]. The replication of the H275Y/I223K mutant virus was significantly reduced compared to that of the corresponding wild-type virus. The H275Y/G147R mutant and the corresponding wild-type viruses had comparable virus titers after $36 \mathrm{~h}$ post-infection; however, the dual mutant virus replicated more efficiently than the wild-type virus during the initial cycle of infection. The G147R substitution confers receptor-binding activity to the NA protein of the $\mathrm{A}(\mathrm{H} 1 \mathrm{~N} 1)$ pdm09 virus [20]. A higher receptor-binding activity may affect faster virus replication at the early stage of infection. These results indicate that the H275Y/I223K substitution negatively affects viral growth, at least in vitro, but that the H275Y/I223R and H275Y/G147R substitutions do not. 


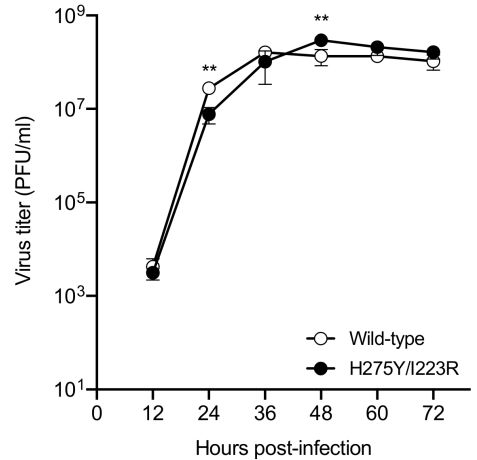

(a)

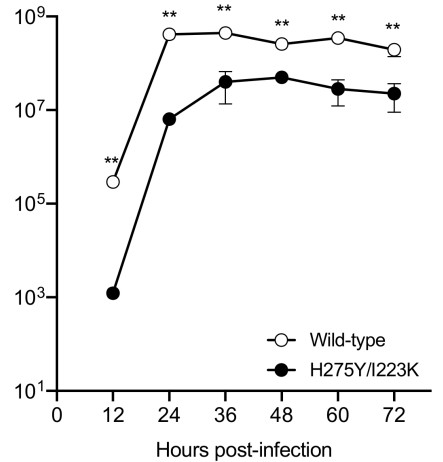

(b)

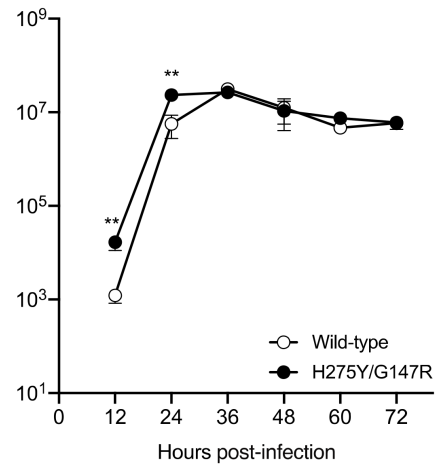

(c)

Figure 1. In vitro replication kinetics of the dual H275Y mutant influenza $A(H 1 N 1) p d m 09$ viruses. AX4 cells were infected with the H275Y/I223R, H275Y/I223K, or H275Y/G147R mutant virus, or the corresponding wild-type virus at a multiplicity of infection of 0.001 plaque-forming units (PFU)/cell in triplicate. The supernatants were harvested at the indicated time and were subjected to virus titration by using plaque assays. Means and standard deviations are shown. Asterisks indicate statistically significant differences between the dual H275Y mutant and wild-type viruses as determined by using unpaired t-test calculated by fitting a mixed-effects model; ${ }^{* *} p<0.01$. Wild-type: (a) A/Sakai/23/2013, (b) A/Yokohama/40/2016, and (c) A/Yokohama/59/2016.

\subsection{Competitive Growth Capabilities of the Dual H275Y Mutant Viruses and the Wild-Type Viruses}

To compare the competitive growth capability of the dual H275Y mutant viruses with that of wild-type viruses, each dual H275Y mutant virus was coinfected with its corresponding wild-type counterpart (Figure 2). The proportion of the H275Y/I223R mutant virus to that of the wild-type virus was similar at passage 1 and then decreased significantly, whereas the proportion of the H275Y/I223K mutant virus to that of the wild-type virus decreased significantly from passage 1 . In contrast, the H275Y/G147R mutant virus rapidly became dominant in the mixed virus populations at passages 1 and 2, indicating that this mutant virus continued to replicate efficiently, competing with the wild-type virus during the initial cycle of infection. These results indicate that the growth capability of the H275Y/G147R virus is comparable to, or somewhat better than, that of the wild-type virus at least in vitro.

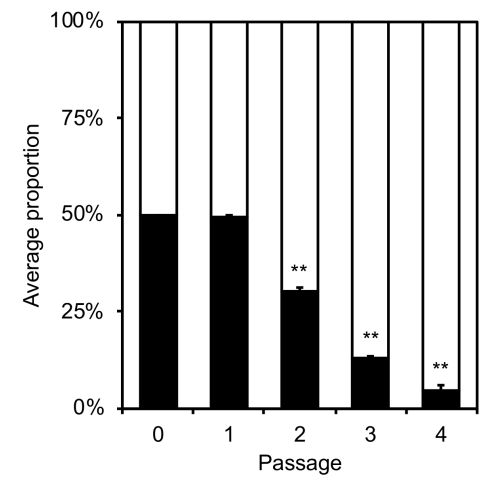

-

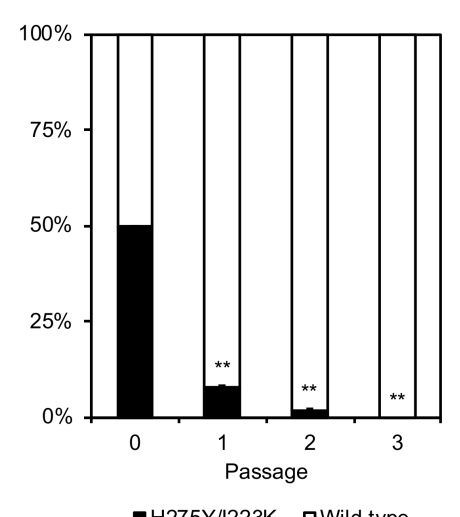

(b)

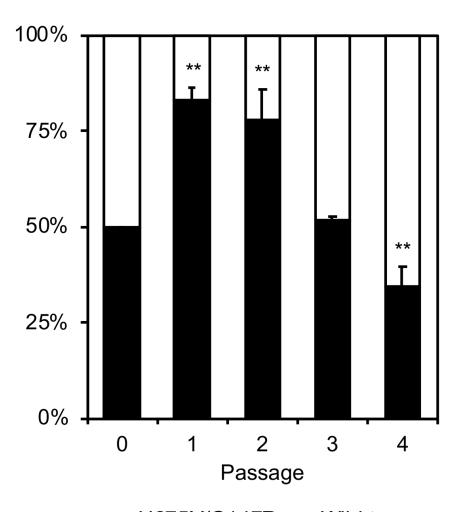

- H275Y/G147R aWild-type

(a)

Figure 2. Competitive growth capabilities of the dual H275Y mutant influenza A(H1N1)pdm09 viruses and the wild-type viruses. AX4 cells were coinfected with the H275Y/I223R, H275Y/I223K, or H275Y/G147R mutant virus and with the corresponding wild-type virus at a multiplicity of infection 
(MOI) of 0.01 plaque-forming units (PFU)/cell in triplicate. At two days post-infection, the supernatants were subjected to virus titration by using plaque assays and to deep sequencing analysis to determine the relative proportion of each genotype. The viruses were serially passaged 3-4 times at an MOI of $0.01 \mathrm{PFU} / \mathrm{cell}$. Means and standard deviations are shown. Asterisks indicate statistically significant differences between the dual H275Y mutant and wild-type viruses as determined by using unpaired t-test calculated by fitting a mixed-effects model; ${ }^{* *} p<0.01$. Wild-type: (a) A/Sakai/23/2013, (b) A/Yokohama/40/2016, and (c) A/Yokohama/59/2016.

\subsection{Effects of Amino Acid Substitutions on the Stability of the NA Protein}

The H275Y substitution caused a detrimental effect on viral fitness by decreasing the stability of the NA protein [21]. Additional substitutions, V241I and N369K, were thought to improve the stability of the NA, thereby compensating for the negative effects of the H275Y substitution on the growth and transmissibility of the mutant virus [22,23]. To assess the effect of the I223R, I223K, and G147R substitutions on the stability of the NA protein, we performed an in silico mutagenesis study as previously described [18] (Figure 3). The changes in stability caused by each of the substitutions I223R, I223K, and G147R were 1.32, 3.29, and $-2.72 \mathrm{kcal} / \mathrm{mol}$, respectively. These data suggest that the I223R and I223K substitutions may destabilize the NA structure, whereas the G147R substitution likely stabilizes NA, consistent with our previous finding [4].

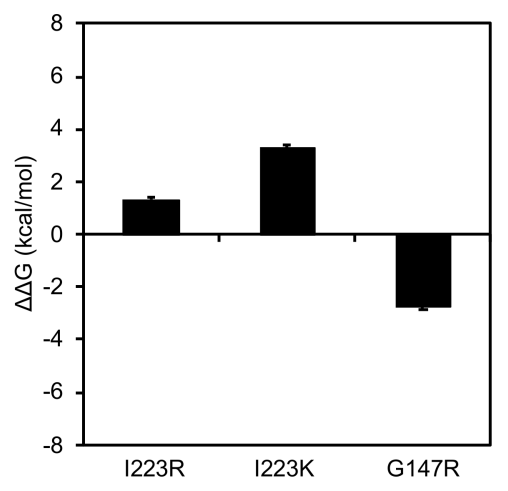

Figure 3. Structural analysis of the influenza A(H1N1)pdm09 virus neuraminidase (NA) protein. Structure models of the NA proteins were constructed by use of homology modeling. Single-point mutations were generated on the NA model. The average stability changes were calculated by using the Boltzmann distribution. Means and standard deviations of the stability scores $(\Delta \Delta \mathrm{Gs})$ of the structures are shown.

\section{Discussion}

Immunocompromised patients are at great risk for emergence of NA inhibitor-resistant viruses [24-27]. The high frequency of resistant viruses among immunocompromised patients is associated with the high levels of viral titers and prolonged viral shedding in these patients [28-31]. $\mathrm{A}(\mathrm{H} 1 \mathrm{N1}) \mathrm{pdm} 09$ viruses carrying the single H275Y substitution rapidly emerge during treatment with oseltamivir and/or peramivir in immunocompromised patients [32,33] and exhibit clinically significant cross-resistance to oseltamivir and peramivir [34]. In the present study, the dual H275Y mutant $\mathrm{A}(\mathrm{H} 1 \mathrm{N1}) \mathrm{pdm} 09$ viruses showed enhanced cross-resistance to oseltamivir and peramivir and reduced susceptibility to zanamivir compared to the single H275Y mutant viruses. Patients infected with the H275Y/I223R or H275Y/I223K mutant virus showed improved clinical and virologic responses after laninamivir treatment, although the laninamivir susceptibilities of these viruses were reduced. The H275Y/G147R mutant virus retained susceptibility to laninamivir, but the patient infected with this mutant virus did not have the opportunity to receive laninamivir treatment before she died. All of the dual H275Y mutant viruses tested were susceptible to baloxavir and favipiravir. Baloxavir 
was approved for the treatment of influenza A and B virus infections in February 2018 in Japan; favipiravir has been approved and stockpiled for use against novel influenza virus infections for which first-line antivirals are ineffective [1]. Therefore, baloxavir could be a treatment option against these multidrug-resistant viruses.

The selective pressure from prolonged exposure to NA inhibitors in immunocompromised patients can lead to the emergence of multidrug resistance. The patients infected with the H275Y/I223K or H275Y/G147R mutant virus received prolonged treatment with oseltamivir and/or peramivir, whereas the patient infected with the H275Y/I223R mutant virus was treated with a single dose of peramivir before specimen collection. A pretreatment specimen from this patient was unavailable, but the specimen collected after four days of peramivir treatment contained the dual H275Y/I223R substitution and not a mixture including wild-type $275 \mathrm{H}$ and 223I. These observations suggest three possibilities: the patient was infected by another host harboring the single H275Y mutant virus, the patient was infected by another host harboring the single I223R mutant virus, or the patient was infected by another host harboring the dual H275Y/I223R mutant virus. Since the NA inhibitor-resistant viruses transmit well in the immunocompromised patients $[35,36]$, we cannot rule out human-to-human transmission of these mutant viruses.

The growth capability of the H275Y/I223K mutant virus was significantly reduced compared to that of the wild-type virus, and the H275Y/I223R mutant virus retained growth capability to some extent. Residue 223 is located within the framework of the NA enzymatic active site [37]. Our structural analysis predicted that the I223K and I223R substitutions destabilize the NA structure, but the stability score for I223K was higher than that for I223R. These results suggest that an NA with the I223K substitution is less stable than an NA with the I223R substitution. Therefore, the H275Y/I223K mutant virus showed reduced viral fitness relative to the H275Y/I223R mutant virus.

A large cluster of the H275Y mutant A(H1N1)pdm09 virus occurred in Sapporo, Japan during the 2013-14 season [18]. This mutant virus emerged prior to the main influenza season and spread predominantly in the community. To understand the reason for this large cluster, we examined the in vitro and in vivo properties of the mutant virus. We found that it grew well in vitro and in vivo, with growth similar to, or somewhat better than, the wild-type virus. However, the mutant NA structure was less stable than that of the wild-type virus. Therefore, once the wild-type virus began to circulate in the community, the mutant virus could not compete and faded out. In the present study, we found that the H275Y/G147R mutant virus retained the ability to grow similarly to, or somewhat better than, the wild-type virus. Residue 147 is located in a 150-loop adjacent to the NA enzymatic active site [4,5]. In our structural analysis, the G147R substitution was predicted to stabilize the NA structure. Therefore, the H275Y/G147R mutant virus retained its viral fitness and might be as transmissible among humans as the wild-type virus. In fact, the patient infected with this mutant virus developed pneumonia without the isolation of bacterial pathogens, suggesting viral pneumonia with this mutant virus [4]. The in vivo properties of this mutant virus should be further investigated.

The H275Y substitution in the $\mathrm{A}(\mathrm{H} 1 \mathrm{~N} 1) \mathrm{pdm} 09$ virus NA protein compromises viral fitness; however, additional V241I and N369K substitutions in the NA protein were reported to increase the replication and transmission fitness of the $\mathrm{H} 275 \mathrm{Y}$ mutant $\mathrm{A}(\mathrm{H} 1 \mathrm{N1}) \mathrm{pdm} 09$ viruses [22,23]. The dual H275Y mutant viruses in the present study possess these permissive substitutions. Furthermore, currently circulating A(H1N1)pdm09 viruses also possess these substitutions, suggesting an increased risk for oseltamivir and peramivir cross-resistant viruses to emerge and spread. The emergence of the multidrug-resistant viruses reduces the antiviral options for treatment. This report highlights the importance of closely monitoring multidrug-resistant viruses in immunocompromised patients to improve clinical management and surveillance of these resistant viruses to protect public health.

Author Contributions: Conceptualization, E.T. and T.O.; analysis and investigation, E.T., S.F., M.Y., M.S., H.M., K.N., N.K., T.K., H.S., I.D., Y.S. (Yuji Sato), S.T., Y.S. (Yukie Shimazu), T.S., T.I., The Influenza Virus Surveillance Group of Japan; writing-original draft preparation, E.T.; writing-review and editing, S.W. and T.O. All authors have read and agreed to the published version of the manuscript. 
Funding: This research was funded by a Grant-in-Aid for Emerging and Reemerging Infectious Diseases from the Ministry of Health, Labour and Welfare, Japan, grant number 10110400 and by Japan Society for the Promotion of Science KAKENHI, grant number JP18K10036.

Acknowledgments: We thank Rie Ogawa, Shiho Nagata, Hideka Miura, Hiromi Sugawara, Miki Akimoto, Aya Sato, and Kayo Watanabe for technical assistance. We also thank Susan Watson for scientific editing. Members of the Influenza Virus Surveillance Group of Japan are Rika Komagome (Hokkaido Institute of Public Health), Asami Ohnishi (Sapporo City Institute of Public Health), Rika Tsutsui (Aomori Prefectural Public Health and Environment Center), Masaki Takahashi (Iwate Prefectural Research Institute for Environmental Sciences and Public Health), Yuko Suzuki (Miyagi Prefectural Institute of Public Health and Environment), Makiko Ushimizu (Sendai City Institute of Public Health), Chihiro Shibata (Akita Prefectural Research Center for Public Health and Environment), Shizuka Tanaka (Yamagata Prefectural Institute of Public Health), Yoshiko Kashiwagi (Fukushima Prefectural Institute of Public Health), Chika Hirokawa (Niigata Prefectural Institute of Public Health and Environmental Sciences), Kazunari Yamamoto (Niigata City Institute of Public Health and Environment), Takako Suzuki (Tochigi Prefectural Institute of Public Health and Environmental Sciences), Shunsuke Kataoka (Utsunomiya City Institute of Public Health and Environment Science), Hiroyuki Tsukagoshi (Gunma Prefectural Institute of Public Health and Environmental Sciences), Noriko Suzuki (Saitama Institute of Public Health), Yuka Uno (Saitama City Institute of Health Science and Research), Noriko Oitate (Chiba Prefectural Institute of Public Health), Wakako Nishikawa (Chiba City Institute of Health and Environment), Sachiko Harada (Tokyo Metropolitan Institute of Public Health), Sumi Watanabe (Kanagawa Prefectural Institute of Public Health), Chiharu Kawakami (Yokohama City Institute of Public Health), Hideaki Shimizu (Kawasaki City Institute of Public Health), Hazime Amano (Yokosuka Institute of Public Health), Sayoko Arakawa (Sagamihara City Institute of Public Health), Masayuki Oonuma (Yamanashi Institute for Public Health), Michiko Takeuchi (Nagano Environmental Conservation Research Institute), Yuichiro Okamura (Nagano City Health Center), Yukiko Sakai (Shizuoka Institute of Environment and Hygiene), Takaharu Maehata (Shizuoka City Institute of Environmental Sciences and Public Health), Toshihiko Furuta (Hamamatsu City Health Environment Research Center), Masatsugu Obuchi (Toyama Institute of Health), Hiroe Kodama (Ishikawa Prefectural Institute of Public Health and Environmental science), Kaori Sato (Fukui Prefectural Institute of Public Health and Environmental Science), Masahiro Nishioka (Gifu Prefectural Research Institute for Health and Environmental Sciences), Yusuke Sato (Gifu Municipal Institute of Public Health), Yoshihiro Yasui (Aichi Prefectural Institute of Public Health), Takuya Yano (Mie Prefecture Health and Environment Research Institute), Hiromi Kodama (Shiga Prefectural Institute of Public Health), Akiko Nagasao (Kyoto City Institute of Health and Environmental Sciences), Satoshi Hiroi and Hideyuki Kubo (Osaka Institute of Public Health), Fumika Okayama (Sakai City Institute of Public Health), Tomohiro Oshibe (Hyogo Prefectural Institute of Public Health and Consumer Sciences), Ai Mori (Kobe Institute of Health), Misako Fujitani (Nara Prefecture Institute of Health), Yuki Matsui (Wakayama Prefectural Research Center of Environment and Public Health), Hidenobu Ekawa (Wakayama City Institute of Public Health), Nobuyuki Kato (Tottori Prefectural Institute of Public Health and Environmental Science), Tetsuo Mita (Shimane Prefectural Institute of Public Health and Environmental Science), Yasuhiro Matsuoka (Okayama Prefectural Institute for Environmental Science and Public Health), Miwako Yamamoto (Hiroshima City Institute of Public Health), Shoichi Toda (Yamaguchi Prefectural Institute of Public Health and Environment), Yumiko Kawakami (Tokushima Prefectural Public Health, Pharmaceutical and Environmental Sciences Center), Yukari Terajima (Kagawa Prefectural Research Institute for Environmental Sciences and Public Health), Akie Ochi (Ehime Prefecture Institute of Public Health and Environmental Science), Noriko Yorimitsu (Kochi Public Health and Sanitation Institute), Yuki Ashizuka (Fukuoka Institute of Health and Environmental Sciences), Shuichi Zaitsu (Fukuoka City Institute of Health and Environment), Takashi Kimura (Kitakyushu City Institute of Health and Environmental Sciences), Katsuyuki Ando (Saga Prefectural Institute of Public Health and Pharmaceutical Research), Kana Miura (Nagasaki Prefectural Institute for Environment Research and Public Health), Kenta Yoshioka (Kumamoto Prefectural Institute of Public-Health and Environmental Science), Kaori Nishizawa (Kumamoto City Environmental Research Center), Miki Kato (Oita Prefectural Institute of Health and Environment), Miho Miura (Miyazaki Prefectural Institute for Public Health and Environment), Yuka Iwamoto (Kagoshima Prefectural Institute for Environmental Research and Public Health), and Yumani Kuba (Okinawa Prefectural Institute of Health and Environment).

Conflicts of Interest: The authors declare no conflict of interest.

\section{References}

1. Takashita, E.; Morita, H.; Ogawa, R.; Nakamura, K.; Fujisaki, S.; Shirakura, M.; Kuwahara, T.; Kishida, N.; Watanabe, S.; Odagiri, T. Susceptibility of influenza viruses to the novel cap-dependent endonuclease inhibitor baloxavir marboxil. Front. Microbiol. 2018, 9, 3026. [CrossRef] [PubMed]

2. Takashita, E.; Ejima, M.; Ogawa, R.; Fujisaki, S.; Neumann, G.; Furuta, Y.; Kawaoka, Y.; Tashiro, M.; Odagiri, T. Antiviral susceptibility of influenza viruses isolated from patients pre- and post-administration of favipiravir. Antivir. Res. 2016, 132, 170-177. [CrossRef] [PubMed]

3. Takashita, E.; Meijer, A.; Lackenby, A.; Gubareva, L.; Rebelo-de-Andrade, H.; Besselaar, T.; Fry, A.; Gregory, V.; Leang, S.K.; Huang, W.; et al. Global update on the susceptibility of human influenza viruses to neuraminidase inhibitors, 2013-2014. Antivir. Res. 2015, 117, 27-38. [CrossRef] [PubMed] 
4. Takashita, E.; Fujisaki, S.; Shirakura, M.; Nakamura, K.; Kishida, N.; Kuwahara, T.; Shimazu, Y.; Shimomura, T.; Watanabe, S.; Odagiri, T.; et al. Influenza a (H1N1) pdm09 virus exhibiting enhanced cross-resistance to oseltamivir and peramivir due to a dual H275Y/G147R substitution, Japan, March 2016. Euro Surveill. 2016, 21. [CrossRef] [PubMed]

5. Gubareva, L.V.; Besselaar, T.G.; Daniels, R.S.; Fry, A.; Gregory, V.; Huang, W.; Hurt, A.C.; Jorquera, P.A.; Lackenby, A.; Leang, S.K.; et al. Global update on the susceptibility of human influenza viruses to neuraminidase inhibitors, 2015-2016. Antivir. Res. 2017, 146, 12-20. [CrossRef] [PubMed]

6. Nguyen, H.T.; Fry, A.M.; Loveless, P.A.; Klimov, A.I.; Gubareva, L.V. Recovery of a multidrug-resistant strain of pandemic influenza a 2009 (H1N1) virus carrying a dual H275Y/I223R mutation from a child after prolonged treatment with oseltamivir. Clin. Infect. Dis. 2010, 51, 983-984. [CrossRef]

7. Hurt, A.C.; Lee, R.T.; Leang, S.K.; Cui, L.; Deng, Y.M.; Phuah, S.P.; Caldwell, N.; Freeman, K.; Komadina, N.; Smith, D.; et al. Increased detection in Australia and Singapore of a novel influenza A(H1N1)2009 variant with reduced oseltamivir and zanamivir sensitivity due to a S247N neuraminidase mutation. Euro Surveill. 2011, 16, 19884. [CrossRef]

8. Nguyen, H.T.; Trujillo, A.A.; Sheu, T.G.; Levine, M.; Mishin, V.P.; Shaw, M.; Ades, E.W.; Klimov, A.I.; Fry, A.M.; Gubareva, L.V. Analysis of influenza viruses from patients clinically suspected of infection with an oseltamivir resistant virus during the 2009 pandemic in the United States. Antivir. Res. 2012, 93, 381-386. [CrossRef]

9. L'Huillier, A.G.; Abed, Y.; Petty, T.J.; Cordey, S.; Thomas, Y.; Bouhy, X.; Schibler, M.; Simon, A.; Chalandon, Y.; van Delden, C.; et al. E119D neuraminidase mutation conferring pan-resistance to neuraminidase Iinhibitors in an a (H1N1) pdm09 isolate from a stem-cell transplant recipient. J. Infect. Dis. 2015, 212, 1726-1734. [CrossRef]

10. Tamura, D.; DeBiasi, R.L.; Okomo-Adhiambo, M.; Mishin, V.P.; Campbell, A.P.; Loechelt, B.; Wiedermann, B.L.; Fry, A.M.; Gubareva, L.V. Emergence of multidrug-resistant influenza a (H1N1) pdm09 virus variants in an immunocompromised child treated with oseltamivir and Zanamivir. J. Infect. Dis. 2015, 212, 1209-1213. [CrossRef]

11. Trebbien, R.; Pedersen, S.S.; Vorborg, K.; Franck, K.T.; Fischer, T.K. Development of oseltamivir and zanamivir resistance in influenza a (H1N1) pdm09 virus, Denmark, 2014. Euro Surveill. 2017, 22, 30445. [CrossRef] [PubMed]

12. LeGoff, J.; Rousset, D.; Abou-Jaoude, G.; Scemla, A.; Ribaud, P.; Mercier-Delarue, S.; Caro, V.; Enouf, V.; Simon, F.; Molina, J.M.; et al. I223R mutation in influenza a (H1N1) pdm09 neuraminidase confers reduced susceptibility to oseltamivir and zanamivir and enhanced resistance with H275Y. PLoS ONE 2012, 7, e37095. [CrossRef] [PubMed]

13. Pizzorno, A.; Abed, Y.; Bouhy, X.; Beaulieu, E.; Mallett, C.; Russell, R.; Boivin, G. Impact of mutations at residue I223 of the neuraminidase protein on the resistance profile, replication level, and virulence of the 2009 pandemic influenza virus. Antimicrob Agents Chemother. 2012, 56, 1208-1214. [CrossRef] [PubMed]

14. Abed, Y.; Bouhy, X.; L'Huillier, A.G.; Rheaume, C.; Pizzorno, A.; Retamal, M.; Fage, C.; Dube, K.; Joly, M.H.; Beaulieu, E.; et al. The E119D neuraminidase mutation identified in a multidrug-resistant influenza a (H1N1) pdm09 isolate severely alters viral fitness in vitro and in animal models. Antivir. Res. 2016, 132, 6-12. [CrossRef]

15. Takada, K.; Kawakami, C.; Fan, S.; Chiba, S.; Zhong, G.; Gu, C.; Shimizu, K.; Takasaki, S.; Sakai-Tagawa, Y.; Lopes, T.J.S.; et al. A humanized MDCK cell line for the efficient isolation and propagation of human influenza viruses. Nat. Microbiol. 2019, 4, 1268-1273. [CrossRef]

16. Hatakeyama, S.; Sakai-Tagawa, Y.; Kiso, M.; Goto, H.; Kawakami, C.; Mitamura, K.; Sugaya, N.; Suzuki, Y.; Kawaoka, Y. Enhanced expression of an alpha2,6-linked sialic acid on MDCK cells improves isolation of human influenza viruses and evaluation of their sensitivity to a neuraminidase inhibitor. J. Clin. Microbiol. 2005, 43, 4139-4146. [CrossRef]

17. Takashita, E.; Abe, T.; Morita, H.; Nagata, S.; Fujisaki, S.; Miura, H.; Shirakura, M.; Kishida, N.; Nakamura, K.; Kuwahara, T.; et al. Influenza a (H1N1) pdm09 virus exhibiting reduced susceptibility to baloxavir due to a PA E23K substitution detected from a child without baloxavir treatment. Antivir. Res. 2020, 180, 104828. [CrossRef] 
18. Takashita, E.; Kiso, M.; Fujisaki, S.; Yokoyama, M.; Nakamura, K.; Shirakura, M.; Sato, H.; Odagiri, T.; Kawaoka, Y.; Tashiro, M. Characterization of a large cluster of influenza a (H1N1) pdm09 viruses cross-resistant to oseltamivir and peramivir during the 2013-2014 influenza season in Japan. Antimicrob. Agents Chemother. 2015, 59, 2607-2617. [CrossRef]

19. Van der Vries, E.; Collins, P.J.; Vachieri, S.G.; Xiong, X.; Liu, J.; Walker, P.A.; Haire, L.F.; Hay, A.J.; Schutten, M.; Osterhaus, A.D.; et al. H1N1 2009 pandemic influenza virus: Resistance of the I223R neuraminidase mutant explained by kinetic and structural analysis. PLoS Pathog. 2012, 8, e1002914. [CrossRef]

20. Hooper, K.A.; Crowe, J.E., Jr.; Bloom, J.D. Influenza viruses with receptor-binding N1 neuraminidases occur sporadically in several lineages and show no attenuation in cell culture or mice. J. Virol. 2015, 89, 3737-3745. [CrossRef]

21. Hurt, A.C.; Hardie, K.; Wilson, N.J.; Deng, Y.M.; Osbourn, M.; Leang, S.K.; Lee, R.T.; Iannello, P.; Gehrig, N.; Shaw, R.; et al. Characteristics of a widespread community cluster of H275Y oseltamivir-resistant a (H1N1) pdm09 influenza in Australia. J. Infect. Dis. 2012, 206, 148-157. [CrossRef] [PubMed]

22. Abed, Y.; Pizzorno, A.; Bouhy, X.; Rheaume, C.; Boivin, G. Impact of potential permissive neuraminidase mutations on viral fitness of the H275Y oseltamivir-resistant influenza A(H1N1)pdm09 virus in vitro, in mice and in ferrets. J. Virol. 2014, 88, 1652-1658. [CrossRef] [PubMed]

23. Butler, J.; Hooper, K.A.; Petrie, S.; Lee, R.; Maurer-Stroh, S.; Reh, L.; Guarnaccia, T.; Baas, C.; Xue, L.; Vitesnik, S.; et al. Estimating the fitness advantage conferred by permissive neuraminidase mutations in recent oseltamivir-resistant $\mathrm{A}(\mathrm{H} 1 \mathrm{~N} 1)$ pdm09 influenza viruses. PLoS Pathog. 2014, 10, e1004065. [CrossRef] [PubMed]

24. Harvala, H.; Gunson, R.; Simmonds, P.; Hardie, A.; Bennett, S.; Scott, F.; Roddie, H.; McKnight, J.; Walsh, T.; Rowney, D.; et al. The emergence of oseltamivir-resistant pandemic influenza a (H1N1) 2009 virus amongst hospitalised immunocompromised patients in Scotland, November-December, 2009. Euro Surveill. 2010, 15, 19536. [CrossRef]

25. Calatayud, L.; Lackenby, A.; Reynolds, A.; McMenamin, J.; Phin, N.F.; Zambon, M.; Pebody, R. Oseltamivir-resistant pandemic (H1N1) 2009 virus infection in England and Scotland, 2009-2010. Emerg. Infect. Dis. 2011, 17, 1807-1815. [CrossRef] [PubMed]

26. Graitcer, S.B.; Gubareva, L.; Kamimoto, L.; Doshi, S.; Vandermeer, M.; Louie, J.; Waters, C.; Moore, Z.; Sleeman, K.; Okomo-Adhiambo, M.; et al. Characteristics of patients with oseltamivir-resistant pandemic (H1N1) 2009, United States. Emerg. Infect. Dis. 2011, 17, 255-257. [CrossRef] [PubMed]

27. Hurt, A.C.; Chotpitayasunondh, T.; Cox, N.J.; Daniels, R.; Fry, A.M.; Gubareva, L.V.; Hayden, F.G.; Hui, D.S.; Hungnes, O.; Lackenby, A.; et al. Antiviral resistance during the 2009 influenza A H1N1 pandemic: Public health, laboratory, and clinical perspectives. Lancet Infect. Dis. 2012, 12, 240-248. [CrossRef]

28. Campanini, G.; Piralla, A.; Rovida, F.; Puzelli, S.; Facchini, M.; Locatelli, F.; Minoli, L.; Percivalle, E.; Donatelli, I.; Baldanti, F.; et al. First case in Italy of acquired resistance to oseltamivir in an immunocompromised patient with influenza A/H1N1v infection. J. Clin. Virol. 2010, 48, 220-222. [CrossRef]

29. Tramontana, A.R.; George, B.; Hurt, A.C.; Doyle, J.S.; Langan, K.; Reid, A.B.; Harper, J.M.; Thursky, K.; Worth, L.J.; Dwyer, D.E.; et al. Oseltamivir resistance in adult oncology and hematology patients infected with pandemic (H1N1) 2009 virus, Australia. Emerg. Infect. Dis. 2010, 16, 1068-1075. [CrossRef]

30. Renaud, C.; Boudreault, A.A.; Kuypers, J.; Lofy, K.H.; Corey, L.; Boeckh, M.J.; Englund, J.A. H275Y mutant pandemic (H1N1) 2009 virus in immunocompromised patients. Emerg. Infect. Dis. 2011, 17, 653-660. [CrossRef]

31. Li, T.C.; Chan, M.C.; Lee, N. Clinical implications of antiviral resistance in influenza. Viruses 2015, 7, 4929-4944. [CrossRef] [PubMed]

32. Gaur, A.H.; Bagga, B.; Barman, S.; Hayden, R.; Lamptey, A.; Hoffman, J.M.; Bhojwani, D.; Flynn, P.M.; Tuomanen, E.; Webby, R. Intravenous zanamivir for oseltamivir-resistant 2009 H1N1 influenza. N. Engl, J. Med. 2010, 362, 88-89. [CrossRef] [PubMed]

33. Renaud, C.; Pergam, S.A.; Polyak, C.; Jain, R.; Kuypers, J.; Englund, J.A.; Corey, L.; Boeckh, M.J. Early emergence of an $\mathrm{H} 275 \mathrm{Y}$ mutation in a hematopoietic cell transplant recipient treated with intravenous peramivir. Transpl. Infect. Dis. 2010, 12, 513-517. [CrossRef] [PubMed]

34. Memoli, M.J.; Hrabal, R.J.; Hassantoufighi, A.; Eichelberger, M.C.; Taubenberger, J.K. Rapid selection of oseltamivir- and peramivir-resistant pandemic H1N1 virus during therapy in 2 immunocompromised hosts. Clin. Infect. Dis. 2010, 50, 1252-1255. [CrossRef] [PubMed] 
35. Chen, L.F.; Dailey, N.J.; Rao, A.K.; Fleischauer, A.T.; Greenwald, I.; Deyde, V.M.; Moore, Z.S.; Anderson, D.J.; Duffy, J.; Gubareva, L.V.; et al. Cluster of oseltamivir-resistant 2009 pandemic influenza a (H1N1) virus infections on a hospital ward among immunocompromised patients-North Carolina, 2009. J. Infect. Dis. 2011, 203, 838-846. [CrossRef] [PubMed]

36. Moore, C.; Galiano, M.; Lackenby, A.; Abdelrahman, T.; Barnes, R.; Evans, M.R.; Fegan, C.; Froude, S.; Hastings, M.; Knapper, S.; et al. Evidence of person-to-person transmission of oseltamivir-resistant pandemic influenza a (H1N1) 2009 virus in a hematology unit. J. Infect. Dis. 2011, 203, 18-24. [CrossRef]

37. Colman, P.M.; Hoyne, P.A.; Lawrence, M.C. Sequence and structure alignment of paramyxovirus hemagglutinin-neuraminidase with influenza virus neuraminidase. J. Virol. 1993, 67, 2972-2980. [CrossRef]

(C) 2020 by the authors. Licensee MDPI, Basel, Switzerland. This article is an open access article distributed under the terms and conditions of the Creative Commons Attribution (CC BY) license (http://creativecommons.org/licenses/by/4.0/). 